\title{
Clostridium difficile A and/or B Toxin Measurement
}

National Cancer Institute

\section{Source}

National Cancer Institute. Clostridium difficile A and/or B Toxin Measurement. NCI

Thesaurus. Code C150858.

The determination of the amount of A and/or B toxin produced by Clostridium difficile present in a sample. 